\title{
Corrigendum
}

\section{Dietary patterns and cardiovascular risk factors in adolescents and young adults: the Northern Ireland Young Hearts Project - CORRIGENDUM}

\author{
Hannah J. McCourt, Claire R. Draffin, Jayne V. Woodside, Chris R. Cardwell, Ian S. Young,
} Steven J. Hunter, Liam J. Murray, Colin A. Boreham, Alison M. Gallagher, Charlotte E. Neville and Michelle C. McKinley* on behalf of the Young Hearts Study Group

(First published online 18 January 2016)

doi:10.1017/S0007114514002682, Published by Cambridge University Press, September 2014.

The blood pressure data that was reported was incorrect. Revised tables of data are below.

In the abstract, the fifth sentence describing the results (page 1685) should be: In the a posteriori DP analysis, YH3 participants adhering most closely to the 'healthy' DP were found to have lower pulse wave velocity (PWV) and homocysteine concentrations, the 'sweet tooth' DP were found to have increased LDL concentrations and decreased HDL concentrations, the 'drinker/social' DP were found to have lower LDL and homocysteine concentrations, but exhibited a trend towards a higher TAG concentration, and finally the 'Western' DP were found to have elevated homocysteine and HDL concentrations.

In the first paragraph of the Results section (page 1688), sentence 5 should be: SBP and DBP increased significantly from YH1 to YH3 $(P<0.001)$ for males, but decreased significantly from YH1 to YH3 $(P<0.05$ and $P<0.001$ respectively) for females.

In the fourth paragraph of the Results section (page 1691), sentences 5 and 6 should be: A high Western dietary pattern score was positively associated with HDL-cholesterol concentrations and homocysteine concentrations in fully adjusted analyses. The fourth and final PCA-derived dietary pattern, sweet tooth, was positively associated with LDL concentrations and inversely associated with HDL concentrations.

The authors apologise for this error.

\section{Reference}

1. Hannah J. McCourt, Claire R. Draffin, Jayne V. Woodside, et al. (2014) Dietary patterns and cardiovascular risk factors in adolescents and young adults: the Northern Ireland Young Hearts Project. Br J Nutr 112, 1685-1698. 
Table 1. General health and lifestyle characteristics and dietary intake in males and females who participated in Young Hearts (YH)1 and YH3 (Mean values and standard deviations)

\begin{tabular}{|c|c|c|c|c|c|c|c|c|c|c|c|c|c|c|c|}
\hline & \multicolumn{5}{|c|}{ Males $(n=249)$} & \multicolumn{5}{|c|}{ Females $(n=238)$} & \multicolumn{4}{|c|}{ All $(n=487)$} & \multirow[b]{3}{*}{$P^{*}$} \\
\hline & \multicolumn{2}{|c|}{$\mathrm{YH} 1$} & \multicolumn{2}{|c|}{ YH3 } & \multirow[b]{2}{*}{$P^{*}$} & \multicolumn{2}{|c|}{ YH1 } & \multicolumn{2}{|c|}{$\mathrm{YH} 3$} & \multirow[b]{2}{*}{$P^{*}$} & \multicolumn{2}{|c|}{$\mathrm{YH} 1$} & \multicolumn{2}{|c|}{$\mathrm{YH} 3$} & \\
\hline & Mean & SD & Mean & SD & & Mean & SD & Mean & SD & & Mean & SD & Mean & SD & \\
\hline Age (years) & 13.9 & 1.5 & $22 \cdot 4$ & 1.6 & $<0.001$ & $14 \cdot 0$ & 1.6 & $22 \cdot 8$ & 1.7 & $<0.001$ & $13 \cdot 9$ & 1.5 & $22 \cdot 6$ & 1.6 & $<0.001$ \\
\hline Height $(\mathrm{m})$ & 1.59 & 0.1 & 1.78 & 0.1 & $<0.001$ & 1.57 & 0.1 & 1.64 & $0 \cdot 1$ & $<0.001$ & 1.58 & 0.1 & 1.71 & 0.1 & $<0.001$ \\
\hline Weight (kg) & 49.5 & 11.1 & $75 \cdot 3$ & 11.5 & $<0.001$ & $50 \cdot 7$ & 11.0 & 64.5 & 11.9 & $<0.001$ & $50 \cdot 1$ & 11.1 & 70.0 & $12 \cdot 9$ & $<0.001$ \\
\hline $\mathrm{BMI}\left(\mathrm{kg} / \mathrm{m}^{2}\right)$ & $19 \cdot 4$ & 2.8 & 23.8 & 3.1 & $<0.001$ & 20.5 & 3.4 & 23.8 & 4.3 & $<0.001$ & 19.9 & 3.1 & 23.8 & 3.7 & $<0.001$ \\
\hline Smoker (n,\%) & 22 & 8.8 & 91 & 36.5 & $<0.001$ & 19 & 8.0 & 86 & $36 \cdot 1$ & 0.002 & 41 & $8 \cdot 4$ & 117 & $36 \cdot 3$ & $<0.001$ \\
\hline Cholesterol $(\mathrm{mmol} / \mathrm{l})$ & 4.4 & 0.8 & 4.5 & 0.9 & 0.243 & 4.7 & 0.8 & 4.7 & 0.9 & 0.273 & 4.5 & 0.8 & 4.6 & 0.9 & 0.109 \\
\hline $\mathrm{HDL}(\mathrm{mmol} / \mathrm{l})$ & 1.3 & 0.3 & 1.3 & 0.3 & $<0.001$ & 1.3 & 0.3 & 1.5 & 0.4 & $<0.001$ & 1.3 & 0.3 & 1.3 & 0.4 & 0.774 \\
\hline SBP $(m m H g)$ & $115 \cdot 4$ & $12 \cdot 9$ & $118 \cdot 7$ & 11.7 & $<0.001$ & 114.0 & $12 \cdot 2$ & $107 \cdot 3$ & $10 \cdot 9$ & $<0.001$ & 114.7 & $12 \cdot 6$ & $113 \cdot 1$ & $12 \cdot 7$ & $<0.05$ \\
\hline $\mathrm{DBP}(\mathrm{mmHg})$ & 69.9 & 9.4 & $76 \cdot 3$ & $9 \cdot 1$ & $<0.001$ & 72.5 & 9.4 & 71.4 & $9 \cdot 3$ & $<0.001$ & $71 \cdot 2$ & 9.5 & 73.9 & 9.5 & $<0.001$ \\
\hline Energy (kcal/d) & 2901.6 & $772 \cdot 0$ & 3121.5 & 852.7 & 0.718 & 2264.4 & 643.8 & 1993.6 & $575 \cdot 3$ & 0.024 & $2590 \cdot 2$ & 779.7 & $2570 \cdot 3$ & 922.5 & 0.665 \\
\hline Total fat $(/ 4.184 \mathrm{~kJ})$ & $42 \cdot 6$ & 4.6 & $36 \cdot 2$ & $6 \cdot 2$ & 0.009 & $43 \cdot 1$ & 5.0 & $36 \cdot 7$ & $6 \cdot 7$ & 0.286 & $42 \cdot 8$ & 4.8 & $36 \cdot 4$ & 6.5 & 0.010 \\
\hline Fibre (/4.184 kJ) & 8.0 & $2 \cdot 3$ & $5 \cdot 7$ & 1.9 & $<0.001$ & 8.7 & $2 \cdot 3$ & $7 \cdot 0$ & 4.4 & $<0.001$ & 8.3 & $2 \cdot 3$ & $6 \cdot 3$ & 3.4 & $<0.001$ \\
\hline MDS & 4.1 & 1.5 & 3.7 & 0.9 & 0.019 & 4.0 & 1.5 & $3 \cdot 7$ & 0.9 & 0.004 & $4 \cdot 1$ & 1.5 & 3.7 & 0.9 & $<0.001$ \\
\hline
\end{tabular}

SBP, systolic blood pressure; DBP, diastolic blood pressure; MDS, Mediterranean Diet Score.

* For each sex, differences between variables measure at $\mathrm{YH} 1$ and $\mathrm{YH} 3$ were assessed using paired sample t-tests and categorical data were assessed by McNemar's test. 
Table 3. CVD risk factors for the five groups of four food patterns determined by a posteriori principal component analysis related to men and women participating in the Young Hearts ( $\mathrm{YH}$ ) 3 (Mean values and standard deviations; adjusted mean values and $95 \%$ confidence intervals)

\begin{tabular}{|c|c|c|c|c|c|c|c|c|c|c|c|c|c|}
\hline \multirow{5}{*}{$\begin{array}{l}\text { Factor } 1 \\
\text { Drinker/ } \\
\text { Social } \\
\text { n-487 }\end{array}$} & \multirow{5}{*}{$\begin{array}{l}\text { Total cholesterol }(\mathrm{mmol} / \mathrm{l}) \\
\text { Full adjustments } \dagger \\
\text { TAG (mmol/l) } \\
\text { Full adjustments } \dagger\end{array}$} & \multicolumn{2}{|c|}{ 1st fifth (lowest intake) } & \multicolumn{2}{|c|}{ 2nd fifth } & \multicolumn{2}{|c|}{3 rd fifth } & \multicolumn{2}{|c|}{ 4th fifth } & \multicolumn{2}{|c|}{ 5th fifth (highest intake) } & \multirow{3}{*}{$\begin{array}{c}P^{*} \text { for trend } \\
0.016 \\
0.118\end{array}$} & \multirow{2}{*}{$\frac{R^{2}}{0.014}$} \\
\hline & & 4.7 & 0.8 & 4.7 & 0.8 & 4.6 & 1.0 & 4.6 & 0.8 & \multirow{2}{*}{\multicolumn{2}{|c|}{$\stackrel{4.3}{\text { Reference }} 0.7$}} & & \\
\hline & & 0.1 & $-0.3,0.5$ & 0.1 & $-0.2,0.5$ & 0.0 & $-0.3,0.3$ & 0.2 & $-0.1,0.5$ & & & & 0.193 \\
\hline & & 0.7 & 0.3 & 0.7 & 0.4 & 0.8 & 0.4 & 0.9 & 0.4 & \multirow{2}{*}{\multicolumn{2}{|c|}{$0.8 \quad 0.5$}} & 0.002 & 0.023 \\
\hline & & -0.2 & $-0.4,0.0$ & -0.2 & $-0.3,0.0$ & -0.1 & $-0.2,0.1$ & 0.0 & $-0.1,0.1$ & & & 0.071 & 0.189 \\
\hline & $\mathrm{HDL}(\mathrm{mmol} / \mathrm{l})$ & 1.5 & 0.4 & 1.4 & 0.4 & $1 \cdot 3$ & 0.3 & $1 \cdot 2$ & 0.3 & \multicolumn{2}{|c|}{$\begin{array}{cc}\text { Reference } & \\
1.3 & 0.3\end{array}$} & $<0.001$ & 0.044 \\
\hline & Full adjustments $\dagger$ & 0.0 & $-0.2,0.1$ & -0.1 & $-0.2,0.1$ & -0.1 & $-0.2,0.0$ & -0.1 & $-0.2,0.0$ & \multicolumn{2}{|c|}{ Reference } & 0.775 & 0.176 \\
\hline & $\mathrm{LDL}(\mathrm{mmol} / \mathrm{l})$ & $2 \cdot 9$ & 0.7 & $3 \cdot 0$ & 0.8 & $2 \cdot 9$ & 1.0 & $3 \cdot 0$ & 0.8 & \multirow{2}{*}{\multicolumn{2}{|c|}{$2.7 \quad 0.8$}} & 0.207 & 0.004 \\
\hline & Full adjustments $\dagger$ & 0.2 & $-0.2,0.6$ & 0.3 & $-0.1,0.6$ & 0.1 & $-0.2,0.4$ & 0.3 & $0.1,0.6$ & Reference & & 0.027 & 0.199 \\
\hline & $\mathrm{SBP}(\mathrm{mmHg})$ & 107.9 & $10 \cdot 8$ & $108 \cdot 6$ & 11.9 & $112 \cdot 4$ & $12 \cdot 0$ & 118.8 & $12 \cdot 0$ & \multicolumn{2}{|c|}{$118.2 \quad 12.6$} & $<0.001$ & 0.118 \\
\hline & Full adjustments $\dagger$ & -4.4 & $-9 \cdot 5,0 \cdot 8$ & -3.8 & $-8.5,0.9$ & -3.6 & $-7 \cdot 5,0.4$ & 0.2 & $-3 \cdot 3,3 \cdot 6$ & Reference & & 0.059 & 0.281 \\
\hline & Full adjustments $\dagger$ & -0.2 & $-3 \cdot 9,4 \cdot 3$ & 0.3 & $-3 \cdot 4,4.0$ & $2 \cdot 0$ & $-1 \cdot 2,5 \cdot 1$ & 3.4 & $0.6,6 \cdot 1$ & Reference & & 0.607 & 0.123 \\
\hline & PWV (ms) & $2 \cdot 9$ & 0.4 & $2 \cdot 9$ & 0.4 & $3 \cdot 2$ & 0.4 & $3 \cdot 2$ & 0.4 & $3 \cdot 3$ & $0 \cdot 6$ & $<0.001$ & 0.125 \\
\hline & Full adjustments $\dagger$ & -0.1 & $-0.3,0.1$ & -0.1 & $-0.2,0.1$ & 0.1 & $-0.1,0.2$ & 0.0 & $-0.2,0.1$ & Reference & & 0.323 & 0.260 \\
\hline & HOMA & 2.5 & $2 \cdot 1$ & $2 \cdot 3$ & 1.5 & 2.5 & 1.2 & 2.5 & 2.0 & $2 \cdot 6$ & $2 \cdot 6$ & 0.531 & 0.001 \\
\hline & Homocysteine $(\mu \mathrm{mol} / \mathrm{l})$ & $9 \cdot 3$ & 3.0 & $10 \cdot 4$ & $5 \cdot 0$ & $10 \cdot 0$ & $5 \cdot 0$ & $10 \cdot 7$ & $6 \cdot 2$ & 9.7 & 4.8 & 0.513 & 0.001 \\
\hline & Full adjustments $\dagger$ & $2 \cdot 2$ & $-0.2,4.5$ & 2.5 & $0.4,4.7$ & 1.5 & $-0.3,3.3$ & 1.4 & $-0.1,3.0$ & Reference & & 0.032 & 0.124 \\
\hline Factor 2 & Total cholesterol $(\mathrm{mmol} / \mathrm{l})$ & 4.5 & 0.8 & 4.7 & 0.9 & 4.6 & 0.8 & 4.7 & 0.9 & 4.4 & 0.9 & 0.723 & 0.723 \\
\hline Healthy & TAG $(\mathrm{mmol} / \mathrm{l})$ & 0.8 & 0.4 & 0.8 & 0.4 & 0.7 & 0.4 & 0.8 & 0.3 & 0.8 & 0.5 & 0.994 & $<0.001$ \\
\hline$n=487$ & $\mathrm{HDL}(\mathrm{mmol} / \mathrm{l})$ & 1.3 & 0.3 & 1.4 & 0.3 & 1.4 & 0.3 & $1 \cdot 3$ & 0.3 & 1.3 & 0.4 & 0.892 & $<0.001$ \\
\hline & $\operatorname{LDL}(\mathrm{mmo} / /)^{\prime}$ & $2 \cdot 9$ & 0.7 & $2 \cdot 9$ & 0.9 & $2 \cdot 8$ & 0.7 & 3.1 & 0.9 & $2 \cdot 7$ & 0.8 & 0.683 & $<0.001$ \\
\hline & $\mathrm{SBP}(\mathrm{mmHg})$ & 114.9 & 13.5 & 113.0 & $12 \cdot 0$ & 111.5 & $12 \cdot 4$ & $112 \cdot 8$ & $12 \cdot 4$ & 113.6 & $13 \cdot 2$ & 0.465 & 0.001 \\
\hline & DBP $(\mathrm{mmHg})$ & $75 \cdot 1$ & $10 \cdot 0$ & 73.7 & 8.9 & 73.5 & 9.4 & 73.8 & $9 \cdot 2$ & 73.2 & $10 \cdot 1$ & 0.204 & 0.003 \\
\hline & HOMA & $2 \cdot \overline{9}$ & 2.8 & $2 \cdot 3$ & 1.2 & $2 \cdot 3$ & 1.4 & $2 \cdot 3$ & 1.1 & $2 \cdot 6$ & $2 \cdot 6$ & 0.324 & 0.003 \\
\hline & Homocysteine $(\mu \mathrm{mol} / \mathrm{l})$ & 11.9 & $7 \cdot 3$ & $10 \cdot 7$ & $5 \cdot 1$ & $9 \cdot 8$ & $4 \cdot 1$ & 9.4 & 4.0 & 8.4 & $2 \cdot 2$ & $<0.001$ & 0.054 \\
\hline & Full adjustments $\dagger$ & 2.6 & $0.8,4.3$ & $2 \cdot 1$ & $0.5,3.7$ & 1.2 & $-0.4,2.8$ & 1.1 & $-0.4,2.7$ & Reference & & 0.004 & 0.134 \\
\hline Factor 3 & Total cholesterol $(\mathrm{mmol} / \mathrm{l})$ & 4.7 & 1.0 & 4.5 & 0.9 & $4 . \overline{6}$ & 0.9 & 4.6 & 0.9 & 4.6 & 0.9 & 0.720 & $<0.001$ \\
\hline Western & TAG $(\mathrm{mmol} / \mathrm{l})$ & 0.8 & 0.4 & 0.8 & 0.4 & 0.8 & 0.3 & 0.8 & 0.4 & 0.8 & 0.4 & 0.951 & $<0.001$ \\
\hline$n=487$ & $\mathrm{HDL}(\mathrm{mmol} / \mathrm{l})$ & 1.3 & 0.3 & $1 \cdot 3$ & 0.3 & 1.4 & 0.4 & 1.4 & 0.3 & 1.3 & 0.4 & 0.328 & 0.002 \\
\hline & Full adjustments $\dagger$ & -0.1 & $-0.2,0.0$ & -0.1 & $-0.2,0.0$ & -0.1 & $-0.2,0.0$ & 0.0 & $-0.2,0.1$ & Reference & & 0.032 & 0.187 \\
\hline & $\mathrm{LDL}(\mathrm{mmol} / \mathrm{l})$ & $3 \cdot 0$ & 0.9 & $2 \cdot 9$ & 0.8 & $2 \cdot 9$ & 0.8 & 2.8 & 0.8 & 2.9 & 0.8 & 0.430 & 0.001 \\
\hline & SBP $(\mathrm{mmHg})$ & $112 \cdot 6$ & $14 \cdot 3$ & $112 \cdot 1$ & 11.5 & $112 \cdot 1$ & 10.9 & 113.8 & $13 \cdot 7$ & 115.2 & $12 \cdot 7$ & 0.095 & 0.006 \\
\hline & Full adjustments $\dagger$ & $-1 \cdot 7$ & $-1 \cdot 7,5 \cdot 0$ & $1 \cdot 1$ & $-2 \cdot 5,4 \cdot 6$ & 0.9 & $-2 \cdot 6,4 \cdot 3$ & 2.8 & $-0.6,6 \cdot 1$ & Reference & & 0.692 & 0.275 \\
\hline & DBP (mmHg) & $72 \cdot 6$ & 8.7 & $73 \cdot 7$ & $10 \cdot 3$ & 73.7 & $8 \cdot 6$ & 73.6 & $10 \cdot 1$ & $75 \cdot 8$ & 9.7 & 0.041 & 0.009 \\
\hline & Full adjustments $\dagger$ & -0.4 & $-3 \cdot 1,2 \cdot 3$ & -0.2 & $-3 \cdot 1,2 \cdot 6$ & 0.3 & $-2 \cdot 4,3 \cdot 1$ & -0.2 & $-2 \cdot 8,2 \cdot 5$ & Reference & & 0.750 & 0.123 \\
\hline & PWV (ms) & 3.1 & 0.4 & $3 \cdot 1$ & 0.6 & $3 \cdot 0$ & 0.4 & $3 \cdot 1$ & 0.4 & $3 \cdot 2$ & 0.4 & 0.152 & 0.005 \\
\hline & HOMA & $2 \cdot 6$ & $2 \cdot 6$ & $2 \cdot 6$ & 2.4 & 2.3 & 1.5 & 2.6 & 1.9 & $2 \cdot 2$ & 1.0 & 0.211 & 0.004 \\
\hline & Homocysteine $(\mu \mathrm{mol} / \mathrm{l})$ & 9.3 & 4.0 & 9.2 & $4 \cdot 0$ & $9 \cdot 1$ & 3.0 & 9.9 & 3.4 & 12.6 & 7.6 & $<0.001$ & 0.044 \\
\hline & Full adjustments $\dagger$ & $-3 \cdot 2$ & $-4 \cdot 8,-1 \cdot 7$ & $-2 \cdot 9$ & $-4 \cdot 5,-1 \cdot 4$ & $-3 \cdot 2$ & $-4.7,-1 \cdot 6$ & -2.5 & $-4 \cdot 0,-1 \cdot 0$ & Reference & & $<0.001$ & 0.161 \\
\hline Factor 4 & Total cholesterol $(\mathrm{mmol} / \mathrm{l})$ & 4.6 & 0.9 & 4.6 & 0.9 & 4.7 & 0.9 & 4.5 & 0.9 & 4.6 & 0.9 & 0.987 & $<0.001$ \\
\hline Sweet & TAG $(\mathrm{mmol} / \mathrm{l})$ & 0.9 & 0.6 & 0.8 & 0.4 & 0.7 & 0.4 & 0.8 & 0.4 & 0.7 & 0.3 & 0.010 & 0.015 \\
\hline Tooth & $\mathrm{HDL}(\mathrm{mmol} / \mathrm{l})$ & 1.3 & 0.4 & 1.4 & 0.4 & 1.4 & 0.4 & 1.3 & 0.3 & 1.3 & 0.3 & 0.224 & 0.003 \\
\hline$n=487$ & Full adjustments $\dagger$ & 0.1 & $0.0,0.2$ & 0.1 & $0.0,0.2$ & 0.1 & $0.0,0.2$ & 0.0 & $-0.1,0.1$ & Reference & & 0.004 & 0.196 \\
\hline & $\mathrm{LDL}(\mathrm{mmol} / \mathrm{l})$ & $2 \cdot 9$ & 0.8 & $2 \cdot 8$ & 0.8 & $2 \cdot 9$ & 0.8 & $2 \cdot 9$ & 0.8 & $2 \cdot 9$ & 0.9 & 0.327 & 0.002 \\
\hline & Full adjustments $\dagger$ & -0.2 & $-0.5,0.0$ & -0.3 & $-0.5,0.0$ & -0.1 & $-0.4,0.1$ & -0.1 & $-0.4,0.1$ & Reference & & 0.008 & 0.204 \\
\hline & $\mathrm{SBP}(\mathrm{mmHg})$ & $114 \cdot 0$ & 13.5 & 111.6 & $13 \cdot 8$ & $112 \cdot 6$ & $12 \cdot 5$ & $113 \cdot 4$ & $10 \cdot 9$ & $114 \cdot 3$ & 12.5 & 0.543 & 0.001 \\
\hline & $\mathrm{DBP}(\mathrm{mmHg})$ & 74.4 & 9.5 & $72 \cdot 6$ & $10 \cdot 0$ & 73.5 & $10 \cdot 2$ & 74.6 & $8 \cdot 7$ & $74 \cdot 3$ & $9 \cdot 1$ & 0.592 & 0.001 \\
\hline
\end{tabular}

SBP, systolic blood pressure; DBP, diastolic blood pressure; PWV, pulse wave velocity; HOMA, homeostatic model assessment. † Fully adjusted for age (years), sex, BMI $\left(\mathrm{kg} / \mathrm{m}^{2}\right)$, social status, physical activity, smoking status and energy (kJ). 
Table 4. CVD risk factors for the three groups using the Mediterranean Diet Score (MDS) in men and women participating in Young Hearts YH 3 (Mean values and standard deviations; adjusted mean values and $95 \%$ confidence intervals)

\begin{tabular}{|c|c|c|c|c|c|c|c|c|}
\hline \multirow[b]{2}{*}{ MDS $(n$ 487) } & \multicolumn{2}{|c|}{$1^{\text {st }}$ third (lowest intake) } & \multicolumn{2}{|c|}{$2^{\text {nd }}$ third } & \multicolumn{2}{|c|}{$3^{\text {rd }}$ third (highest intake) } & \multirow[t]{2}{*}{$P$ for trend } & \multirow[t]{2}{*}{$\mathbf{R}^{2}$} \\
\hline & & & & & & & & \\
\hline Total cholesterol $(\mathrm{mmol} / \mathrm{l})$ & 4.7 & 0.9 & 4.5 & 0.8 & 4.6 & 1.0 & 0.475 & 0.001 \\
\hline TAG (mmol/l) & 0.7 & 0.3 & 0.8 & 0.4 & 0.8 & 0.4 & 0.197 & 0.004 \\
\hline $\mathrm{HDL}(\mathrm{mmol} / \mathrm{l})$ & 1.4 & 0.3 & $1 \cdot 3$ & 0.3 & 1.4 & 0.4 & 0.757 & $<0.001$ \\
\hline LDL (mmol/l) & $2 \cdot 9$ & 0.8 & $2 \cdot 9$ & 0.8 & $2 \cdot 8$ & 0.9 & 0.366 & 0.022 \\
\hline SBP (mmHg) & 113.5 & $12 \cdot 8$ & $113 \cdot 7$ & $13 \cdot 0$ & $112 \cdot 3$ & $12 \cdot 3$ & 0.372 & 0.002 \\
\hline DBP $(\mathrm{mmHg})$ & 74.1 & $9 \cdot 0$ & $73 \cdot 3$ & 9.5 & 74.1 & $10 \cdot 1$ & 0.945 & $<0.001$ \\
\hline PWV (ms) & $3 \cdot 1$ & 0.5 & $3 \cdot 1$ & 0.4 & $3 \cdot 1$ & 0.5 & 0.259 & 0.003 \\
\hline Full adjustments $\dagger$ & 0.1 & $0 \cdot 0,0 \cdot 2$ & 0.0 & $0.0,0.2$ & \multicolumn{2}{|c|}{ Reference } & 0.089 & 0.264 \\
\hline HOMA & $2 \cdot 4$ & 1.4 & $2 \cdot 6$ & $2 \cdot 6$ & $2 \cdot 4$ & 1.9 & 0.737 & $<0.001$ \\
\hline Homocysteine $(\mu \mathrm{mol} / \mathrm{l})$ & 9.9 & 3.8 & $10 \cdot 3$ & 5.9 & 9.9 & $5 \cdot 1$ & 0.935 & $<0.001$ \\
\hline
\end{tabular}

SBP, systolic blood pressure; DBP, diastolic blood pressure; PWV, pulse wave velocity; HOMA, homeostatic model assessment.

* Data were analysed using linear regression (unadjusted, adjusted and further adjusted as described with CVD risk factors as the outcome and dietary patterns in tertiles as a

continuous variable).
† Fully adjusted for age (years), sex, BMI $\left(\mathrm{kg} / \mathrm{m}^{2}\right)$, social status, physical activity, smoking status and energy (kJ).

Table 5. Change in Mediterranean Diet Score (MDS) from Young Hearts (YH) 1 to YH3 and CVD risk biomarkers at young adulthood (YH3) (Mean values and standard deviations; adjusted mean values and $95 \%$ confidence intervals)

\begin{tabular}{|c|c|c|c|c|c|c|c|c|c|}
\hline \multirow{3}{*}{$\frac{\text { YH3 CVD risk markers }}{\mathrm{BMI}\left(\mathrm{kg} / \mathrm{m}^{2}\right)}$} & \multicolumn{8}{|c|}{ Change in MDS from YH1 - YH3 } & \multirow{3}{*}{$\frac{P^{*}}{0 \cdot 160}$} \\
\hline & \multicolumn{2}{|c|}{$\begin{array}{c}\text { Least adherent } 1 \text { - least } \\
\text { adherent } 3\end{array}$} & \multicolumn{2}{|c|}{$\begin{array}{l}\text { Least adherent } 1 \text { - most } \\
\text { adherent } 3\end{array}$} & \multicolumn{2}{|c|}{$\begin{array}{c}\text { Most adherent } 1 \text { - least } \\
\text { adherent } 3\end{array}$} & \multicolumn{2}{|c|}{$\begin{array}{c}\text { Most adherent } 1 \text { - most } \\
\text { adherent } 3\end{array}$} & \\
\hline & 23.9 & 3.9 & $23 \cdot 3$ & 3.3 & 24.4 & 3.7 & 24.5 & 3.9 & \\
\hline Waist circumference $(\mathrm{cm})$ & 78.5 & $10 \cdot 8$ & $77 \cdot 1$ & 9.4 & 79.9 & $10 \cdot 1$ & $80 \cdot 4$ & $10 \cdot 8$ & 0.218 \\
\hline Cholesterol (mmo/l) & 4.6 & 0.8 & 4.5 & 1.0 & $4 \cdot 7$ & 1.0 & 4.7 & 1.0 & 0.661 \\
\hline TAG $(\mathrm{mmol} / \mathrm{l})$ & 0.8 & 0.4 & 0.8 & 0.5 & 0.9 & 0.5 & 0.8 & 0.3 & 0.425 \\
\hline $\mathrm{HDL}(\mathrm{mmo} / \mathrm{l})$ & 1.4 & 0.3 & 1.4 & 0.4 & 1.3 & 0.3 & 1.3 & 0.4 & 0.233 \\
\hline LDL (mmol/l) & 2.9 & 0.8 & $2 \cdot 7$ & 0.8 & 3.0 & 0.9 & $3 \cdot 1$ & 0.9 & 0.157 \\
\hline $\mathrm{SBP}(\mathrm{mmHg})$ & 113.4 & $12 \cdot 0$ & $112 \cdot 4$ & $10 \cdot 2$ & $116 \cdot 9$ & $16 \cdot 3$ & 111.5 & 11.7 & 0.461 \\
\hline $\mathrm{DBP}(\mathrm{mmHg})$ & 74.2 & 9.4 & $74 \cdot 8$ & 9.8 & 74.2 & 9.7 & 74.8 & 11.0 & 0.987 \\
\hline PWV (ms) & $3 \cdot 1$ & 0.4 & $3 \cdot 1$ & 0.5 & $3 \cdot 1$ & 0.5 & $3 \cdot 1$ & 0.4 & 0.791 \\
\hline HOMA & $2 \cdot 6$ & $2 \cdot 1$ & $2 \cdot 6$ & $2 \cdot 1$ & $2 \cdot 1$ & 0.7 & 1.8 & 0.9 & 0.139 \\
\hline Homocysteine $(\mu \mathrm{mol} / \mathrm{l})$ & 9.9 & 4.5 & 9.6 & 4.5 & $11 \cdot 4$ & $6 \cdot 4$ & $10 \cdot 8$ & $6 \cdot 6$ & 0.151 \\
\hline Full adjustmentst & -2.5 & $-4.4,-0.6$ & -2.3 & $-4 \cdot 3,-0.3$ & -0.3 & $-2 \cdot 7,2 \cdot 1$ & \multicolumn{2}{|c|}{ Reference } & 0.009 \\
\hline
\end{tabular}

SBP, systolic blood pressure; DBP, diastolic blood pressure; PWV, pulse wave velocity; HOMA, homeostatic model assessment.

* Data analysed using multivariable regression analysis (unadjusted, adjusted and further adjusted as described. 
Table 6. Change in the Mediterranean Diet Score (MDS) from Young Hearts (YH) 1 to $\mathrm{YH} 3$ and changes in CVD risk biomarkers from YH1 to YH3

(Mean values and standard deviations)

\begin{tabular}{|c|c|c|c|c|c|c|c|c|c|}
\hline \multirow{3}{*}{$\begin{array}{l}\text { Change in cardiovascular risk markers } \\
\text { from } \mathrm{YH} 1 \text { to } \mathrm{YH} 3 \dagger\end{array}$} & \multicolumn{8}{|c|}{ Change in MDS from YH1 to YH3 } & \multirow[b]{3}{*}{$P^{*}$} \\
\hline & \multicolumn{2}{|c|}{$\begin{array}{l}\text { Least adherent YH1- least } \\
\text { adherent YH3 }\end{array}$} & \multicolumn{2}{|c|}{$\begin{array}{l}\text { Least adherent YH1- most } \\
\text { adherent YH3 }\end{array}$} & \multicolumn{2}{|c|}{$\begin{array}{l}\text { Most adherent YH1- least } \\
\text { adherent YH3 }\end{array}$} & \multicolumn{2}{|c|}{$\begin{array}{l}\text { Most adherent YH1- most } \\
\text { adherent YH3 }\end{array}$} & \\
\hline & Mean & SD & Mean & SD & Mean & SD & Mean & SD & \\
\hline BMI $\left(\mathrm{kg} / \mathrm{m}^{2}\right)^{\star}$ & 4.0 & 3.0 & 3.8 & 2.6 & 3.7 & 3.0 & 3.5 & 3.0 & 0.688 \\
\hline Cholesterol $(\mathrm{mmo} /)^{*}$ & 0.0 & 0.8 & 0.2 & 0.9 & 0.1 & 0.9 & 0.1 & 0.9 & 0.559 \\
\hline $\mathrm{HDL}\left(\mathrm{mmo} / \mathrm{l}^{*}\right.$ & 0.0 & 0.3 & 0.0 & 0.4 & 0.0 & 0.3 & 0.0 & 0.4 & 0.482 \\
\hline $\mathrm{SBP}(\mathrm{mmHg})^{\star}$ & $-1 \cdot 6$ & $17 \cdot 1$ & $-4 \cdot 3$ & 13.7 & $-1 \cdot 2$ & $16 \cdot 2$ & $-4 \cdot 0$ & $11 \cdot 2$ & 0.708 \\
\hline $\mathrm{DBP}(\mathrm{mmHg})^{*}$ & 4.0 & 11.3 & 1.3 & 9.5 & 2.4 & 12.7 & 1.1 & 11.5 & 0.516 \\
\hline
\end{tabular}

SBP, systolic blood pressure; DBP, diastolic blood pressure.

* Data analysed using multivariable regression analysis (unadjusted, adjusted and further adjusted as described).

† Change calculated as $\mathrm{YH} 3$ values minus $\mathrm{YH} 1$ values. 